\title{
Secret Sharing Schemes for Very Dense Graphs`
}

\author{
Amos Beimel $^{1}$, Oriol Farràs ${ }^{2}$, and Yuval Mintz ${ }^{1}$ \\ 1 Ben Gurion University of the Negev, Be'er Sheva, Israel \\ 2 Universitat Rovira i Virgili, Tarragona, Spain \\ \{beimel, yuvalmin\}@cs.bgu.ac.il, oriol.farras@urv.cat
}

\begin{abstract}
A secret-sharing scheme realizes a graph if every two vertices connected by an edge can reconstruct the secret while every independent set in the graph does not get any information on the secret. Similar to secret-sharing schemes for general access structures, there are gaps between the known lower bounds and upper bounds on the share size for graphs. Motivated by the question of what makes a graph "hard" for secret-sharing schemes, we study very dense graphs, that is, graphs whose complement contains few edges. We show that if a graph with $n$ vertices contains $\left(\begin{array}{c}n \\ 2\end{array}\right)-n^{1+\beta}$ edges for some constant $0 \leq \beta<1$, then there is a scheme realizing the graph with total share size of $\tilde{O}\left(n^{5 / 4+3 \beta / 4}\right)$. This should be compared to $O\left(n^{2} / \log n\right)$ - the best upper bound known for general graphs. Thus, if a graph is "hard", then the graph and its complement should have many edges. We generalize these results to nearly complete $k$-homogeneous access structures for a constant $k$. To complement our results, we prove lower bounds for secret-sharing schemes realizing very dense graphs, e.g., for linear secret-sharing schemes we prove a lower bound of $\Omega\left(n^{1+\beta / 2}\right)$.
\end{abstract}

\section{Introduction}

A secret-sharing scheme, introduced by 94331, is a method by which a dealer, which holds a secret string, can distribute strings, called shares, to a set of participants, enabling only predefined subsets of participants to reconstruct the secret from their shares. The collection of predefined subsets authorized to reconstruct the secret is called the access structure. We consider perfect schemes, in which any unauthorized set of participants should learn nothing about the secret from their combined shares. Secret-sharing schemes are useful cryptographic building blocks, used in many secure protocols, e.g., multiparty computation [717 19], threshold cryptography [24], access control [39], attribute-based encryption [30.51, and oblivious transfer 44,50.

For a scheme to be efficient and be useful for the above mentioned applications, the size of the shares should be small (i.e., polynomial in the number of participants). There are access structures that have efficient schemes, e.g., the threshold access structure, in which the authorized sets are all sets containing at least $\ell$ participants (for some threshold $\ell$ ) 943]. For every access structure

\footnotetext{
^ This work was supported by ISF grant 938/09.
} 
there exist secret-sharing schemes realizing it 31. However, the best known schemes for general access structures, e.g., 84513 34, are highly inefficient, that is, for most access structures the size of shares is $2^{O(n)}$, where $n$ is the number of parties in the access structure. The best lower bound known on the total share size for an explicit or implicit access structure is $\Omega\left(n^{2} / \log n\right)$. Thus, there exists a large gap between the known upper and lower bounds. Bridging this gap is one of the most important questions in the study of secret-sharing schemes. We lack sufficient methods for proving lower bounds on the share size. Furthermore, we lack the sufficient understanding of which access structures are "hard", that is, which access structures require large shares (if any). In contrast to general secret-sharing schemes, super-polynomial lower bounds are known for linear secret-sharing schemes, that is, for schemes where the shares are generated using a linear transformation. It was proved that there exists an explicit access structure such that the total share size of any linear secret-sharing scheme realizing it is $n^{\Omega(\log n)} 32829$. Linear secret-sharing schemes are important as most known secret-sharing schemes are linear and many cryptographic applications require that the scheme is linear.

In this paper we consider a special family of access structures, in which all minimal authorized sets are of size 2 . These access structures can be described by a graph, where each participant is represented by a vertex and each minimal authorized set is represented by an edge. Graph access structures are useful and interesting and have been studied in, e.g., [10/2 14|21|22 23|25|37/47/49]. Many of the results found for graph access structures, using graph theory, were later extended to apply to all access structure. This is illustrated by the next example.

Example 1. Blundo et al. [12] proved that the share size of graph access structures is either the size of the secret or at least 1.5 times larger than that size. This was generalized later to many other families of access structures. MartíFarré and Padró 38 . proved that the share size of every access structure that is not matroidal is at least 1.5 times larger than the size of the secret.

Other results on graph access structures have been extended to homogeneous access structures 36 4146], which are access structures whose minimal authorized subsets are of the same size, and access structures described by simple hypergraphs 2048 .

Every graph access structure can be realized by a scheme in which the total share size is $O\left(n^{2} / \log n\right)$ 151126]; this scheme is linear. The best lower bound for the total share size required to realize a graph access structure by a general secret-sharing scheme is $\Omega(n \log n)[251021$. The best lower bound for the total share size required to realize a graph access structure by a linear secret-sharing scheme is $\Omega\left(n^{3 / 2}\right)$ [6]. Although the gap between the lower and upper bounds is smaller than that of general access structures, studying this gap might reveal new insight that could be applied to the share size of general access structures.

Our Results. In this work we study a natural family of graphs - very dense graphs. These are graphs that have $\left(\begin{array}{l}n \\ 2\end{array}\right)-\ell$ edges for $\ell \ll n^{2}$ (where $n$ is the number of vertices in the graph). The motivation for this work is trying to 
understand which graphs are "hard", that is, which graphs require total share size of $\Omega\left(n^{2} / \operatorname{polylog} n\right.$ ) (if any). For example, if a graph contains $\ell$ edges, then it can be realized by a trivial secret-sharing in which the total share size is $2 \ell$ times the size of the secret 31. Thus, if there exists a "hard" graph then it has to have $\Omega\left(n^{2} /\right.$ polylog $\left.n\right)$ edges. We are interested in the question if these "hard" graphs can be very dense. Our results show that this is not possible.

Our main result is that if a graph has $\left(\begin{array}{l}n \\ 2\end{array}\right)-n^{1+\beta}$ edges for some $0 \leq \beta \leq 1$, then it can be realized by a secret-sharing scheme in which the total share size is $\tilde{O}\left(n^{5 / 4+3 \beta / 4}\right) 1$ this scheme is linear. In particular, if $\beta$ is a constant smaller than 1 , the total share size is $\ll n^{2}$, that is, these are not "hard" graphs as discussed above. Similarly, if $\beta<1 / 3$, then the share size is $o\left(n^{3 / 2}\right)$; thus, these graphs are easier than the graphs for which [6] proved their lower bounds for linear secret-sharing schemes. As a corollary of our main result we prove that if a graph has $\left(\begin{array}{l}n \\ 2\end{array}\right)-\ell$ edges, where $\ell<n / 2$, then it can be realized by a scheme in which the share size is $n+O\left(\ell^{5 / 4}\right)$. Thus, if $\ell \ll n^{4 / 5}$, then the total share size is $n+o(n)$, which is optimal up to an additive factor of $o(n)$.

We extend the techniques used in this result to the study of two additional problems. First, we consider the following scenario: we start with a graph and remove few edges from it. The question is how much the share size of a secretsharing scheme realizing the graph can grow as a result of the removed edges. If we add edges, then trivially the share size grows at most linearly in the number of added edges. We show that also when removing edges, the share size does not increase too much. We study this problem also for general access structures, considering the removal of minimal authorized subsets for any access structure. We show that for certain access structures the share size does not increase too much either. Second, we study the removal of $\ell$ minimal authorized subsets from $k$-out-of- $n$ threshold access structures. We present a construction with total share size $\tilde{O}(\ell n)$ for $k \ll n$.

To complement our results, we prove lower bounds on the share size of secretsharing schemes realizing very dense graphs. For graph access structures, the known lower bounds for general secret-sharing schemes 251021 and linear secret-sharing schemes [6] use sparse graphs with $\theta(n \log n)$ edges and $\theta\left(n^{3 / 2}\right)$ edges, respectively. Using the above lower bounds, we prove lower bounds of $\Omega(\beta n \log n)$ and $\Omega\left(n^{1 / 2+\beta / 2}\right)$ for general and linear secret-sharing schemes respectively for graphs with $\left(\begin{array}{l}n \\ 2\end{array}\right)-n^{1+\beta}$ edges. In addition, we prove lower bounds of $n+\ell$ for graphs with $\left(\begin{array}{l}n \\ 2\end{array}\right)-\ell$ edges, where $\ell<n / 2$. Our lower bounds are not tight, however, they prove, as can be expected, that for linear secretsharing schemes the total share size grows as a function of the number of excluded edges. The lower bounds for linear schemes are interesting as most known secret-sharing schemes, including the schemes constructed in this paper, are linear.

Techniques. Brickell and Davenport 14 proved that a connected graph has an ideal scheme (that is, a scheme in which the total share size is $n$ times the size

${ }^{1}$ We use the $\tilde{O}$ notation which ignores polylogarithmic factors. 
of the secret) if and only if the graph is a complete multipartite graph 2 To construct a scheme realizing a very dense graph, we cover the graph by complete multipartite graphs (in particular, cliques), that is, we construct a sequence of multipartite graphs $G_{1}, G_{2}, \ldots, G_{r}$ such that each graph $G_{i}$ is a subgraph of $G$ and each edge of $G$ is an edge in at least one graph $G_{i}$. We next, for every $i$, share the secret independently using the ideal secret-sharing scheme realizing $G_{i}$. The total share size in the resulting scheme is the sum of the number of vertices in the graphs $G_{1}, G_{2}, \ldots, G_{r}$. This idea of covering a graph was used in previous schemes, e.g., [11]2. The contribution of this paper is how to find a "good" cover for every dense graph.

Our starting point is constructing a scheme for graphs in which every vertex is adjacent to nearly all other vertices, that is, graphs where the degree of every vertex in the complement graph is bounded by some $d \ll n$. We cover such graphs by equivalence graphs, that is, graphs which are union of disjoint cliques. Alon 1 proved, using a probabilistic proof, that every such graph can be covered by $O\left(d^{2} \log n\right)$ equivalence graphs. We improve on this result, and prove, using a different probabilistic proof, that every such graph can be covered by $O(d \log n)$ equivalence graphs. The total share size of the resulting scheme is $\tilde{O}(d n)$.

We use the above scheme to realize very dense graphs. We first cover all vertices whose degree in the complement graph is "big". There are not too many such vertices in the complement graph, and the share size in realizing each star (namely, a vertex and its adjacent edges) is at most $n$. Once we removed all edges adjacent to vertices whose degree is "big", we use the cover by equivalence graphs to cover the remaining edges. To achieve a better scheme, we first remove vertices of high degree using stars, then use covers of bipartite graphs of [33] to further reduce the degree of the vertices in the complement graph, and finally use the cover by equivalence graphs.

Additional Related Work. Sun and Shieh [48] consider access structures that are defined by a forbidden graph, where each party is represented by a vertex, and 2 parties are an unauthorized set iff their vertices are connected by an edge. They give a construction which had an information ratio of $n / 2$. In 48, every set of size 3 can reconstruct the secret. Our problem is much harder as every independent set in the graph is unauthorized.

\section{Preliminaries}

In this section we define secret-sharing schemes and provide some background material used in this work. We present a definition of secret-sharing as given in [1815].

Definition 2. Let $P=\left\{p_{1}, \ldots, p_{n}\right\}$ be a set of parties. A collection $\Gamma \subseteq 2^{P}$ is monotone if $B \in \Gamma$ and $B \subseteq C$ imply that $C \in \Gamma$. An access structure is a monotone collection $\Gamma \subseteq 2^{P}$ of non-empty subsets of $P$. Sets in $\Gamma$ are called

\footnotetext{
${ }^{2}$ See Section 2 for the graph terminology used in the rest of this section.
} 
authorized, and sets not in $\Gamma$ are called unauthorized. The family of minimal authorized subsets is noted by $\min \Gamma$.

$A$ distribution scheme $\Sigma=\langle\Pi, \mu\rangle$ with domain of secrets $K$ is a pair, where $\mu$ is a probability distribution on some finite set $R$ called the set of random strings and $\Pi$ is a mapping from $K \times R$ to a set of $n$-tuples $K_{1} \times K_{2} \times \cdots \times K_{n}$, where $K_{j}$ is called the domain of shares of $p_{j}$. A dealer distributes a secret $k \in K$ according to $\Sigma$ by first sampling a random string $r \in R$ according to $\mu$, computing a vector of shares $\Pi(k, r)=\left(s_{1}, \ldots, s_{n}\right)$, and privately communicating each share $s_{j}$ to party $p_{j}$. For a set $A \subseteq P$, we denote $\Pi(s, r)_{A}$ as the restriction of $\Pi(s, r)$ to its A-entries. The (normalized) total share size of a distribution scheme is $\sum_{1 \leq j \leq n} \log \left|K_{j}\right| / \log |K|$.

Definition 3 (Secret Sharing). Let $K$ be a finite set of secrets, where $|K| \geq 2$. $A$ distribution scheme $\langle\Pi, \mu\rangle$ with domain of secrets $K$ is a secret-sharing scheme realizing an access structure $\Gamma$ if the following two requirements hold:

Correctness. The secret $k$ can be reconstructed by any authorized set of parties.

PRIVACY. Every unauthorized set cannot learn anything about the secret (in the information theoretic sense) from their shares.

In this work we mainly consider graph access structures. Let $G=(V, E)$ be an undirected graph. We consider the graph access structure, where the parties are the vertices of the graph and the minimal authorized sets are the edges. In other words, a set of vertices can reconstruct the secret iff it contains an edge. In the rest of the paper we will not distinguish between the graph and the access structure it describes and we will not distinguish between vertices and parties.

Next we define the graph terminology that we use throughout this paper. The degree of a graph is the maximum degree of vertices in a graph. A graph $G^{\prime}=\left(V^{\prime}, E^{\prime}\right)$ is a subgraph of a graph $G=(V, E)$ if $V^{\prime} \subseteq V$ and $E^{\prime} \subseteq E$. A $k$-partite graph $G=\left(V_{1}, \ldots, V_{k}, E\right)$, where $V_{1}, \ldots, V_{k}$ are disjoint, is a graph whose vertices are $\cup_{i=1}^{k} V_{k}$ such that if $(u, v) \in E$, then there are $i \neq j$ such that $u \in V_{i}$ and $v \in V_{j}$ (that is, there are edges only between vertices in different parts). A $k$-partite graph is complete if it contains all edges between vertices in different parts. A graph is a multipartite graph if it is $k$-partite for some $k$. For example, a clique is a complete $k$-partite graph, where $k$ is the number of vertices in the clique. A bipartite graph in which $\left|V_{1}\right|=1$ is called a star; the vertex in $V_{1}$ is the center and the ones in $V_{2}$ are the leaves.

Brickell and Davenport [14] proved that a connected graph can be realized by an ideal scheme (that is, by a scheme with total share size $n$ ) iff the graph is a complete multipartite graph. As we use the ideal scheme for multipartite graphs we describe it below.

Theorem 4 ([14]). Let $G=\left(V_{1}, \ldots, V_{k}, E\right)$ be a complete multipartite graph and $p>k$ be a prime. There is a linear secret-sharing realizing $G$ where the domain of secrets and the domain of shares of each party are $\{0, \ldots, p-1\}$. 
Proof. Let $s \in\{0, \ldots, p-1\}$ be the secret. We first generate shares in Shamir's 2 -out-of- $k$ scheme [43] for the secret $s$. That is, we choose $a \in\{0, \ldots, p-1\}$ at random with uniform distribution and we compute the share $s_{i}=a \cdot i+s \bmod p$ for $1 \leq i \leq k$. Next, we give $s_{i}$ to all vertices in $V_{i}$. Two vertices from different parts, say $V_{i}$ and $V_{j}$, can reconstruct the secret as follows: $s=\left(j s_{i}-i s_{j}\right) /(j-i)$ (where the arithmetic is in $\mathbb{F}_{p}$ - the finite field with $p$ elements).

Remark 5. The total share size in the above scheme is $n$. However, it requires that $p>k$. In the rest of the paper we assume that $p>n$, thus, we can realize every multipartite subgraph of a graph $G$ with $n$ vertices. This is a reasonable requirement that assumes that the number of bits in the secret is at least $\log n$. We will not mention the size of the secret in the rest of the paper and only consider the total share size of the scheme.

In the rest of the paper we will construct schemes, where we choose subgraphs of $G$ which are multipartite, and share the secret $s$ independently for each subgraph. The following is a well-known lemma.

Lemma 6. Let $G=(V, E)$ be a graph and $G_{1}=\left(V_{1}, E_{1}\right), \ldots, G_{r}=\left(V_{r}, E_{r}\right)$ be subgraphs of $G$ such that each $G_{i}$ is a complete multipartite graph and $E=$ $\cup_{i=1}^{r} E_{i}$ (that is, $G_{1}, \ldots, G_{r}$ cover $G$ ). Assume that we share a secret $s$ independently for each $G_{i}$ using the multipartite scheme. Then, the resulting scheme realizes $G$.

Description of the Problem. In this work we study the problem of realizing a graph access structure, where the graph has few excluded edges. Specifically, let $G=(V, E)$ be an undirected graph with $|V|=n$ and $|E|=\left(\begin{array}{l}n \\ 2\end{array}\right)-\ell$ for some $0<\ell<\left(\begin{array}{l}n \\ 2\end{array}\right)$. We consider the complement graph $\bar{G}=(V, \bar{E})$, where $e \in \bar{E}$ iff $e \notin E$. We call $\bar{G}$ the excluded graph and call its edges the excluded edges. In the rest of the paper, the excluded graph $\bar{G}$ is a sparse graph with $\ll\left(\begin{array}{c}n \\ 2\end{array}\right)$ edges.

Example 7. Assume $\ell=1$, that is, there is one excluded edge, say $\left(v_{n-1}, v_{n}\right)$. In this case, the graph can be realized by an ideal scheme as the graph is the complete $(n-1)$-partite graph, where $v_{n-1}, v_{n}$ are in the same part.

Example 8. Assume $\ell=2$, and there are two adjacent excluded edges, say $\left(v_{n-2}, v_{n}\right)$ and $\left(v_{n-1}, v_{n}\right)$. In this case, the graph $G$ is not a complete multipartite graph, hence it cannot be realized by an ideal scheme [14. However, it can be realized by a scheme in which each of the parties $v_{1}, \ldots, v_{n-3}, v_{n}$ gets a share whose size is the size of the secret and $v_{n-2}, v_{n-1}$ get a share whose size is twice the size of the secret. The scheme is as follows: Generate shares according to the Shamir's 2-out-of- $(n-2)$ secret-sharing scheme, and give party $v_{i}$ the $i$ th share in Shamir's scheme for $1 \leq i \leq n-2$. In addition give to $v_{n-1}$ and $v_{n}$ the $(n-2)$ th share in Shamir's scheme. Using the above shares every pair of parties, except for pairs contained in $\left\{v_{n-2}, v_{n-1}, v_{n}\right\}$, can reconstruct the secret. As the only authorized pair in $\left\{v_{n-2}, v_{n-1}, v_{n}\right\}$ is $\left(v_{n-2}, v_{n-1}\right)$, we give them additional shares: we choose two random strings $r_{1}$ and $r_{2}$ whose exclusive-or is the secret, and give $r_{1}$ to $v_{n-2}$ and $r_{2}$ to $v_{n-1}$. By [11, the total size of shares to realize $G$ is at least $n+2$. That is, the above scheme is optimal. 


\section{Constructions for Bounded Degree Excluded Graphs}

If the excluded graph contains few edges, then the average degree of its vertices is small. We first construct a scheme for graphs such that the degree of all vertices in its excluded graph is bounded by some $d$. In Section 4 we show how we can use this construction for any graph with few excluded edges.

The construction of a secret-sharing scheme for a graph $G$ whose excluded graph $\bar{G}$ has bounded degree uses a cover of $G$ by cliques such that each vertex is contained in a relatively small number of cliques. This is useful as cliques have an ideal scheme. To construct this cover we use colorings of the excluded graph.

Definition 9. An equivalence graph is a vertex-disjoint union of cliques. An equivalence cover of $G=(V, E)$ is a collection of equivalence graphs $G_{1}=$ $\left(V, E_{1}\right), \ldots, G_{r}=\left(V, E_{r}\right)$, each of them is a subgraph of $G$ (that is, $E_{i} \subseteq E$ ), such that this collection covers all the edges of $G$ (that $i s, \cup_{1 \leq i \leq r} E_{i}=E$ ).

$A$ coloring of a graph $\bar{G}=(V, \bar{E})$ with c colors is a mapping $\mu: V \rightarrow\{1, \ldots, c\}$ such that $\mu(u) \neq \mu(v)$ for every $(u, v) \in \bar{E}$.

Lemma 10. Let $G=(V, E)$ be a graph such that the degree of every vertex in its excluded graph $\bar{G}$ is at most d. Then there exists an equivalence cover of $G$ with $r=16 d \ln n$ equivalence graphs.

Proof. An equivalence cover of $G$ can be described by a coloring of $\bar{G}$ and vice versa: given a coloring $\mu$ of $\bar{G}$ we construct an equivalence graph $G^{\prime}=\left(V, E^{\prime}\right)$, which is a subgraph of $G$, where two vertices in $G^{\prime}$ are connected if they are colored by the same color, that is, $E^{\prime}=\{(u, v): \mu(u)=\mu(v)\}$. For every color, the set of vertices colored by such color is an independent set in $\bar{G}$, hence a clique in $G$.

The existence of an equivalence cover of $G$ of size $r$ is proved by using the probabilistic method (see, e.g., [2]). We choose $r$ random colorings $\mu_{1}, \ldots, \mu_{r}$ of $\bar{G}$ with $4 d$ colors. That is, each coloring is chosen independently with uniform distribution among all colorings of $\bar{G}$ with $4 d$ colors. For every coloring $\mu_{i}$, we consider the equivalence graph $G_{i}$ as described above. We next prove that with probability at least half $G_{1}, \ldots, G_{r}$ is an equivalence cover of $G$.

Let $(u, v) \in E$. We first fix $i$ and compute the probability that $u$ and $v$ have the same color in the random coloring $\mu_{i}$. Fix an arbitrary coloring of all vertices except for $u$ and $v$. We prove that conditioned on this coloring, the probability that $u$ and $v$ are colored in the same color is at least $1 /(8 d)$ : The number of colors not used by the neighbors of $u$ and $v$ is at least $2 d$, thus, the probability that $u$ is colored by such color is at least half, and the probability that in this case $v$ is colored in the same color as $u$ is at least $1 /(4 d)$. That is, with probability at least $1 /(8 d)$, the edge $(u, v)$ is covered by the graph $G_{i}$.

The probability that an edge $(u, v)$ is not covered by the $r$ random equivalence graphs $G_{1}, \ldots, G_{r}$ is at most $(1-1 / 8 d)^{r} \leq e^{-r / 8 d}=1 / n^{2}$. Thus, the probability that there exists an edge $(u, v) \in E$ that is not covered by the $r$ random equivalence graphs $G_{1}, \ldots, G_{r}$ is at most $\left(\begin{array}{c}n \\ 2\end{array}\right) / n^{2}<1 / 2$. In particular, such cover with $r$ equivalence graphs exists. 
Remark 11. The existence of the equivalence cover in Lemma 10 is not constructive as we need to choose a random coloring of a graph of bounded degree. Such coloring can be chosen with nearly uniform distribution in polynomial time using a Markov process 3242. Given a collection of equivalence graphs, it is easy to check that for every edge $(u, v) \in E$ there is at least one graph in the collection that covers $(u, v)$. If this is not the case we repeat the process of choosing $r$ random colorings until we find a good collection. The expected number of collections of colorings that have to be chosen before finding a good one is $O(1)$. Thus, we get a randomized polynomial-time algorithm to construct the equivalence cover.

Alon 1] observed that the size of the smallest equivalence cover of a graph $G$ is smaller than the smallest clique cover of $G$. He further proved that if the degree of every vertex in $\bar{G}$ is at most $d$, then $G$ can be covered by $O\left(d^{2} \ln n\right)$ cliques. We directly analyze the size of the smallest equivalence cover and get an equivalence cover of size $O(d \ln n)$. To the best of our knowledge such bound was not known prior to our work.

Lemma 12. Let $G=(V, E)$ be a graph such that the maximum vertex degree in $\bar{G}=(V, \bar{E})$ is less or equal to $d$. Then, $G$ can be realized by a secret-sharing scheme in which the total share size is $\tilde{O}(n d)$.

Proof. Consider a collection of $r=16 d \ln n$ equivalence graphs that cover $G$ (as guaranteed by Lemma 10). We realize the access structure of each equivalence graph $G_{i}$ in the collection by an ideal scheme: For every clique $C$ in $G_{i}$, generate shares in Shamir's 2-out-of- $|C|$ secret-sharing scheme, and distribute the shares among the parties of $C$.

For every excluded edge $(u, v) \notin E$, the vertices $u$ and $v$ are in different cliques in each $G_{i}$ (as $G_{i}$ is a subgraph of $G$ ). Thus, in the above scheme $u$ and $v$ do not get any information. On the other hand, every edge $(u, v) \in E$ is covered by at least one graph $G_{i}$, that is, $u$ and $v$ are in the clique in $G_{i}$, thus, $u$ and $v$ can reconstruct the secret. As in each graph $G_{i}$ each party gets one share, the total share size of the resulting scheme is $n r=O(d n \ln n)=\tilde{O}(n d)$.

We can save a factor of $O(\ln n)$ by using an equivalence cover of size $O(d \ln n)$ such that each edge $(u, v) \in E$ is covered by $O(\ln n)$ graphs in the cover (the existence of such cover can be proved by the same arguments as in the proof of Lemma 10] using a Chernoff bound). We then use Stinson decomposition techniques [47, to construct a scheme with total share size $O(n d)$. The details will be explained in the full version of the paper.

\subsection{Constructions for Bipartite Graphs with Bounded Degree}

As a step in constructing a secret-sharing scheme realizing a graph with few excluded edges, we will need to realize certain bipartite graphs. In this section we show how to realize them using bipartite covers. 
Definition 13 (Complete-bipartite cover and bipartite complement). Let $H=(U, V, E)$ be a bipartite graph. A complete-bipartite cover of $H=$ $(U, V, E)$ is a collection of complete bipartite graphs $H_{1}=\left(U_{1}, V_{1}, E_{1}\right), \ldots, H_{r}=$ $\left(U_{r}, V_{r}, E_{r}\right)$ (that is $E_{i}=U_{i} \times V_{i}$ ), each of them is a subgraph of $H$, such that this collection covers all the edges of $H$ (that is, $\cup_{1 \leq i \leq r} E_{i}=E$ ).

The bipartite complement of a graph $H$ is the bipartite graph $\bar{H}=(U, V, \bar{E})$, where $(u, v) \in \bar{E}$ iff $(u, v) \notin E$ for every $u \in U$ and $v \in V$.

Note that the bipartite complement of a bipartite graph is a bipartite graph and it differs from the complement of the bipartite graph.

Lemma 14 (Jukna [33, Theorem 1]). Let $H=(U, V, E)$ be a bipartite graph such that $|U| \leq|V|$ and the degree of every vertex in $V$ in the bipartite complement graph $\bar{H}$ is at most d. Then there exists a cover of $H$ with $O(d \ln n)$ complete bipartite graphs, where $|V|=n$.

Lemma 15. Let $d<n$ and $H=(U, V, E)$ be a bipartite graph such that $|U|=k$, $|V|=n \geq k$, and the degree of every vertex in $U$ in $\bar{H}$ is at most $d$. Then, $H$ can be realized by a secret-sharing scheme in which the total share size is $\tilde{O}\left(n+k^{3 / 2} d\right)$. If $k=(n / d)^{2 / 3}$, the total share size is $\tilde{O}(n)$.

Proof. Let $D=\left\{v \in V: \exists_{u \in U}\right.$ such that $\left.(u, v) \in \bar{E}\right\}$. As the degree of every vertex in $U$ in $\bar{H}$ is at most $d$, the size of $D$ is at most $d k$. Furthermore, the complete bipartite graph $H_{1}=(U, V \backslash D, U \times(V \backslash D))$ is a subgraph of $H$. We realize $H_{1}$ by an ideal scheme in which the total share size is less than $|U|+|V|=O(n)$.

Now, define $D_{2}=\{v \in D:$ The degree of $v$ in $\bar{H}$ is at least $\sqrt{k}\}$. As $\bar{H}$ contains at most $d k$ edges, $\left|D_{2}\right| \leq d \sqrt{k}$. Let $H_{2}=\left(U, D_{2}, E \cap\left(U \times D_{2}\right)\right)$. The number of edges in $H_{2}$ is less than $|U|\left|D_{2}\right| \leq k^{3 / 2} d$, thus, we can realize $H_{2}$ by secret-sharing scheme in which the total share size is $O\left(k^{3 / 2} d\right)$.

Finally, let $V_{3}=D \backslash D_{2}$ and $H_{3}=\left(U, V_{3}, E \cap\left(U \times V_{3}\right)\right)$. The degree of each vertex in $V_{3}$ in the graph $\overline{H_{3}}$ is at most $\sqrt{k}$, thus, by Lemma 14, $H_{3}$ can be covered by $r=O(\sqrt{k} \ln n)$ complete bipartite graphs. We realize each such bipartite graph by an ideal scheme in which the total share size is $|U|+\left|V_{3}\right| \leq$ $k+k d=O(k d)$. Thus, we realize $H_{2}$ by a scheme in which the total share size is $O(r k d)=O\left(k^{3 / 2} d \ln n\right)$. As $H_{1}, H_{2}$, and $H_{3}$ cover $H$, we constructed a scheme realizing $H$ in which the total share size is $\tilde{O}\left(n+k^{3 / 2} d\right)$. Taking $k=(n / d)^{2 / 3}$, the total share size is $\tilde{O}(n)$.

\section{Constructions for Excluded Graph with Few Edges}

We next show how to use the schemes of Lemma 12 and Lemma 15 to realize excluded graphs with $\ell=n^{1+\beta}$ edges, where $0 \leq \beta<1$. We will start with a simple approach and then use more complicated constructions to achieve better upper bounds. We construct our scheme in steps, where in each step: (1) We choose a set of vertices $V^{\prime} \subseteq V$. (2) We give shares to the parties in $V^{\prime}$ and the 
rest of the parties, such that each edge adjacent to a party in $V^{\prime}$ can reconstruct the secret, and all other pairs of parties (i.e., unauthorized pairs containing parties in $V^{\prime}$ and all pairs not adjacent to $V^{\prime}$ ) get no information on the secret. (3) We remove the vertices in $V^{\prime}$ and all their adjacent edges from the graph. We repeat the following step until all vertices in $\bar{G}$ have small degree and then use the equivalence covering scheme of Section 3 to realize the remaining graph. In this process we will ensure that the total share size remains relatively small. In the following, $n$ will always refer to the number of vertices in the original graph.

Our first step is removing all vertices whose degree in $\bar{G}$ is "high".

Lemma 16. Let $G$ be a graph such that its excluded graph $\bar{G}$ contains at most $n^{1+\beta}$ edges, where $0 \leq \beta<1$. Then, for every $d<n$, we can give shares of size $O\left(n^{2+\beta} / d\right)$ and remove a set of vertices from $G$ and all adjacent edges and obtain an induced subgraph $G^{\prime}$ of $G$ such that $\overline{G^{\prime}}$ contains at most $n^{1+\beta}$ edges and the degree of $\overline{G^{\prime}}$ is at most $d$.

Proof. We choose a vertex $v$ whose degree in $\bar{G}$ is greater than $d$ and consider the star whose center is $v$ and its leaves are all neighbors of $v$ in $G$. We realize this star using an ideal scheme and remove $v$ and its adjacent edges from $G$. The total share size in this step is at most $n$.

We choose another vertex whose degree in $\bar{G}$ is greater than $d$ and do the same until no vertices with degree greater than $d$ exist in $\bar{G}$. As in the beginning there are $n^{1+\beta}$ edges in $\bar{G}$ and in each step we remove at least $d$ edges from $\bar{G}$, the number of steps is at most $n^{1+\beta} / d$. Thus, the total share size of the resulting scheme for the removed vertices is $O\left(n n^{1+\beta} / d\right)$.

We can combine the constructions of Lemma 16] and Lemma 12. That is, we choose some $d \leq n$, remove vertices with degree higher than $d$ in $\bar{G}$, and then apply the equivalence cover construction to the remaining graph $G$, where the degree of $\bar{G}$ is $d$. Thus, the total share size of the resulting scheme (including the scheme from of Lemma 12) is $\tilde{O}\left(n^{2+\beta} / d+d n\right)$. To minimize the share size we take $d=\sqrt{n^{1+\beta}}$ and get a scheme in which the total share size is $\tilde{O}\left(n^{1.5+\beta / 2}\right)$.

Using Lemma 16 we decrease the degrees of the vertices in $\bar{G}$. Instead of applying the construction of Lemma 12 to the resulting graph, we will apply some intermediate steps to further reduce the degree and only then use the construction of Lemma 12 .

Lemma 17. Let $\alpha^{\prime}<\alpha \leq 1$ such that $\alpha \geq 0.25$ and $G=(V, E)$ be a graph such that the degree of $\bar{G}$ is at most $n^{\alpha}$ and $\bar{G}$ contains $\ell$ edges. Then, we can remove a set of vertices and all adjacent edges from the graph and obtain a graph $G^{\prime}$ such that the degree of $\overline{G^{\prime}}$ is at most $n^{\alpha^{\prime}}$, the graph $\overline{G^{\prime}}$ contains $\ell-\ell^{\prime}$ excluded edges for some $\ell^{\prime}>0$, and the total share size for the removed edges is $\tilde{O}\left(\ell^{\prime} n^{1 / 3+2 \alpha / 3-\alpha^{\prime}}\right)$.

Proof. Let $d=n^{\alpha}$ and $d^{\prime}=n^{\alpha^{\prime}}$. We remove the vertices of degree larger than $d^{\prime}$ in steps. In each step we choose an arbitrary set $F$ of $k=(n / d)^{2 / 3}$ vertices of degree at least $d^{\prime}$ in $\bar{G}$ (if the number of vertices of degree $d^{\prime}$ is smaller than $k$, then take the remaining vertices of degree $d^{\prime}$ and put them in $F$ ). Consider all 
edges between vertices of $F$, there are less than $k^{2}=n^{4 / 3} / d^{4 / 3} \leq n$ such edges (since $\left.d \geq n^{1 / 4}\right)$. Next consider the bipartite graph $H=(F, V \backslash F, E \cap(F \times(V \backslash$ $F))$ ). By Lemma 15, we can realize $H$ with a scheme in which the total share size is $O(n)$. Thus, we can remove the vertices in $F$ and all edges adjacent to them, and the total share size in the scheme for every step is $\tilde{O}(n)$.

Let $\ell^{\prime}$ the total number of edges we removed from $\bar{G}$ in these steps until the degree of $\bar{G}$ is at most $d^{\prime}$. As each vertex we remove has degree at least $d^{\prime}$ in $\bar{G}$, the number of vertices we remove is at most $\ell^{\prime} / d^{\prime}$. In each step, except for the last, we remove a set $F$ with $(n / d)^{2 / 3}$ vertices, thus, the number of sets we remove is at most $1+\ell^{\prime} /\left(d^{\prime}(n / d)^{2 / 3}\right)=O\left(\ell^{\prime} d^{2 / 3} /\left(d^{\prime} n^{2 / 3}\right)\right)$. As in each step the share size is $\tilde{O}(n)$, the total share size for the edges we removed from $G$ is $\tilde{O}\left(\ell^{\prime} n^{1 / 3} d^{2 / 3} / d^{\prime}\right)=\tilde{O}\left(\ell^{\prime} n^{1 / 3+2 \alpha / 3-\alpha^{\prime}}\right)$.

We next show how to construct secret-sharing schemes for graphs with few excluded edges using the three building blocks presented so far: (1) initial degree reductions using stars, (2) $O(\log \log n)$ steps of degree reduction using complete bipartite graphs and stars, and (3) using the equivalence cover construction on the graph with reduced degree.

Theorem 18. Let $G=(V, E)$ be a graph with $|V|=n$ and $|E|=\left(\begin{array}{l}n \\ 2\end{array}\right)-n^{1+\beta}$ for some $0 \leq \beta<1$. There exists a secret-sharing scheme realizing $G$ with total share size $\tilde{O}\left(n^{5 / 4+3 \beta / 4}\right)$.

Proof. Let $\alpha_{0}$ be a constant to be determined later. We first apply Lemma 16 with $d=n^{\alpha_{0}}$ and obtain a graph $G$ such that the degree of $\bar{G}$ is at most $d$. The total share size in this step is

$$
O\left(n^{2+\beta} / d\right)=O\left(n^{2+\beta-\alpha_{0}}\right) .
$$

Next define $\alpha_{i}=\left(3-2(2 / 3)^{i}\right) \alpha_{0}-2+2(2 / 3)^{i}$ for $1 \leq i \leq \log \log n$. We choose these constants such that $2 \alpha_{i} / 3-\alpha_{i+1}=2 / 3-\alpha_{0}$. We next repeatedly apply the degree reduction of Lemma 17, we apply it $\log \log n$ times. In the $i$ th invocation of the lemma, where $0 \leq i<\log \log n$, we take $\alpha=\alpha_{i}$ and $\alpha^{\prime}=\alpha_{i+1}$. The cost of each invocation is $\tilde{O}\left(\ell_{i} n^{1 / 3+2 \alpha_{i} / 3-\alpha_{i+1}}\right)=\tilde{O}\left(\ell_{i} n^{1-\alpha_{0}}\right)$, where $\ell_{i}$ is the number of edges removed from $\bar{G}$ in the $i$ th invocation. As the number of edges removed in all invocations is at most $n^{1+\beta}$, the total share size in all these invocations is

$$
\tilde{O}\left(n^{1+\beta} n^{1-\alpha_{0}}\right)=\tilde{O}\left(n^{2+\beta-\alpha_{0}}\right) .
$$

After the $\log \log n$ invocations of Lemma 17. the degree of each vertex in $\bar{G}$ is at most $n^{\alpha_{\log \log n}}=O\left(n^{3 \alpha_{0}-2}\right)$. In the final stage we use Lemma 12 and realize the graph with total share size

$$
\tilde{O}\left(n n^{3 \alpha_{0}-2}\right)=\tilde{O}\left(n^{3 \alpha_{0}-1}\right) .
$$

The total share of realizing $G$ (by (1), (2) , and (3) $)$ is $O\left(n^{2+\beta-\alpha_{0}}\right)+\tilde{O}\left(n^{2+\beta-\alpha_{0}}\right)+$ $\tilde{O}\left(n^{3 \alpha_{0}-1}\right)$. To minimize this expression, we require that $2+\beta-\alpha_{0}=3 \alpha_{0}-1$, thus, $\alpha_{0}=3 / 4+\beta / 4$ and the total share size in the scheme is $\tilde{O}\left(n^{5 / 4+3 \beta / 4}\right)$. 
It can be checked that the construction of the cover of $G$ by multipartite graphs, as done in the above scheme, can be done by a probabilistic algorithm in expected polynomial time. Thus, the computation of the dealer and the parties in our scheme is efficient. In Theorem 18 we showed how to realize a graph where the number of excluded edges is small, however it is at least $n$. We next show how to realize graphs where the number of excluded edges is less than $n$.

Corollary 19. Let $G=(V, E)$ be a graph with $|V|=n$ and $|E|=\left(\begin{array}{l}n \\ 2\end{array}\right)-\ell$ for some $\ell<n / 2$. There exists a secret-sharing scheme realizing $G$ with total share size $n+\tilde{O}\left(\ell^{5 / 4}\right)$.

Proof. Let $V^{\prime} \subseteq V$ be the set of vertices adjacent to excluded edges. As there are $\ell$ excluded edges, the size of $V^{\prime}$ is at most $2 \ell$. Without loss of generality, let $V=\left\{v_{1}, \ldots, v_{n}\right\}$ and $V^{\prime}=\left\{v_{t}, \ldots, v_{n}\right\}$ for some $t>n-2 \ell$. We first execute Shamir's 2-out-of- $t$ secret-sharing scheme and give the share $s_{i}$ to party $v_{i}$ for $1 \leq i<t$, and give the share $s_{t}$ to $v_{i}$ for $t \leq i \leq n$.

Let $V^{\prime \prime}$ be such that $V^{\prime} \subseteq V^{\prime \prime}$ and $\left|V^{\prime \prime}\right|=2 \ell$. Furthermore, let $G^{\prime}=\left(V^{\prime \prime}, E^{\prime}\right)$ be the subgraph of $G$ induced by $V^{\prime \prime}$. The graph $G^{\prime}$ has at most $n^{\prime}=2 \ell$ vertices and $\ell \leq n^{\prime}$ excluded edges, thus, by Theorem 18 (with $\beta=0$ ), it can be realized by a scheme in which the total share size is $\tilde{O}\left(\ell^{5 / 4}\right)$. The total share size in realizing $G$ is, therefore, $n+\tilde{O}\left(\ell^{5 / 4}\right)$.

\section{Constructions for Homogeneous Access Structures}

In this section we extend the techniques used in the construction of graph secretsharing schemes to the construction of schemes for homogeneous access structures, which are access structures whose minimal authorized subsets are of the same size. Every $k$-homogeneous access structure has a monotone formula of size $O\left(n^{k} / \log n\right)($ see [52, Theorem 7.3]), thus, by [8], it can be realized by a secret-sharing scheme with total share size $O\left(n^{k} / \log n\right)$. Other upper bounds for hypergraphs are presented in 36414648]; however they are useful for sparse access structures. In this section, we present constructions for dense homogeneous access structures for a constant $k$. We will describe these access structures by hypergraphs.

A hypergraph is a pair $H=(V, E)$ where $V$ is a set of vertices and $E \subseteq 2^{V} \backslash\{\emptyset\}$ is the set of hyperedges. In this work we consider hypergraphs in which no hyperedge properly contains any other hyperedge. A hypergraph is $k$-uniform if $|e|=k$ for every $e \in E$. A $k$-uniform hypergraph is complete if $E=\left(\begin{array}{l}V \\ k\end{array}\right)=\{e \subseteq V$ : $|e|=k\}$. For any $k$-uniform hypergraph we define the complement hypergraph $\bar{H}=(V, \bar{E})$, with $\bar{E}=\left(\begin{array}{l}V \\ k\end{array}\right) \backslash E$. Observe that there is a one-to-one correspondence between uniform hypergraphs and homogeneous access structures, and that complete hypergraphs are in correspondence with threshold access structures.

By analogy to graphs, we define an equivalence k-hypergraph as a vertexdisjoint union of complete $k$-uniform hypergraphs, and the equivalence cover of a $k$-uniform hypergraph $H=(V, E)$ as a collection of equivalence $k$-hypergraphs 
$H_{1}=\left(V, E_{1}\right), \ldots, H_{r}=\left(V, E_{r}\right)$ with $E_{i} \subseteq E$ for $i=1, \ldots, r$ and $\cup_{1 \leq i \leq r} E_{i}=E$. A weak coloring with $c$ colors of a hypergraph $H=(V, E)$ is a mapping $\mu: V \rightarrow$ $\{1, \ldots, c\}$ such that for every $e \in E$ there exist $u, v \in e$ with $\mu(u) \neq \mu(v)$.

Lemma 20. Let $H=(V, E)$ be a k-uniform hypergraph such that the degree of every vertex in its excluded hypergraph is at most $d$. Then there exists an equivalence cover of $H$ with $r=2^{k} k^{k} d^{k-1} \ln n$ equivalence hypergraphs.

The proof of this lemma is analogous to the proof of Lemma 10, In this case, the result is obtained by using $r$ weak colorings of $\bar{H}$ with $2 k d$ colors.

Lemma 21. Let $H=(V, E)$ be a $k$-uniform hypergraph such that the maximum vertex degree of $\bar{H}=(V, \bar{E})$ is less or equal to $d$. There exists a secret-sharing scheme realizing $H$ in which the total share size is $\tilde{O}\left(2^{k} k^{k} d^{k-1} n\right)$.

Proof. Take the equivalence cover of $H$ of size $r=2^{k} k^{k} d^{k-1} \ln n$ guaranteed by Lemma 20. Now we realize each equivalence hypergraph $H_{i}$ in the collection by an ideal scheme: For every complete hypergraph $C$ in $H_{i}$, generate shares in Shamir's $k$-out-of- $|C|$ secret-sharing scheme. Using arguments similar to the ones used in the proof of Lemma 12, this scheme realizes $H$ and the total share size of the resulting scheme is $n r=\tilde{O}\left(2^{k} k^{k} d^{k-1} n\right)$.

In Theorem 23, we construct a secret-sharing scheme for every excluded hypergraph with few edges. For this purpose, we use a recursive argument based on the construction illustrated in the following example.

Example 22. Let $H=(V, E)$ be a hypergraph and let $v \in V$ be a vertex satisfying that $v \in e$ for every $e \in E$. Consider the hypergraph $H^{\prime}=\left(V^{\prime}, E^{\prime}\right)$ with $V^{\prime}=V \backslash\{v\}$ and $E^{\prime}=\{e \backslash\{v\}: e \in E\}$. If there exists a secret-sharing scheme realizing $H^{\prime}$ with total share size $r$, then we can construct a scheme realizing $H$ with total share size $r+1$ as follows. In order to share a secret $s$, the dealer chooses at random $s_{1}$ and $s_{2}$ satisfying $s=s_{1}+s_{2}$, sends $s_{1}$ to $v$, and shares $s_{2}$ among $V^{\prime}$ using the scheme realizing $H^{\prime}$.

Theorem 23. Let $H=(V, E)$ be a k-hypergraph with $|V|=n$ and $|E|=\left(\begin{array}{l}n \\ k\end{array}\right)-$ $n^{1+\beta}$ for some $0 \leq \beta<k-1$. There exists a secret-sharing scheme realizing $H$ with total share size $\tilde{O}\left(2^{k} k^{k} n^{2+\beta}\right)$.

Proof. By induction on $k$, we prove that for every $H=(V, E)$ satisfying the hypothesis there exists a secret-sharing scheme with total share size $\tilde{O}\left(2^{k} k^{k} \ell^{1-\varepsilon_{k}} n\right)$, where $\ell=n^{1+\beta}$ and $\varepsilon_{k}$ is defined by the equation $\varepsilon_{i+1}=\frac{\varepsilon_{i}}{i+\varepsilon_{i}}$ and $\varepsilon_{1}=1$. By Theorem 18 this property is satisfied for $k=2$. Let $H=(V, E)$ be a $k$ hypergraph with $k>2$. Define $d=\ell^{\frac{1}{k-1+\varepsilon_{k-1}}}$.

We choose a vertex $v$ adjacent to $\ell_{1}>d$ excluded hyperedges. By the hypothesis, there is a secret sharing scheme with total share size $\tilde{O}\left(2^{k-1}(k-\right.$ $\left.1)^{k-1} \ell_{i}^{1-\varepsilon_{k-1}} n\right)$ for the $(k-1)$-hypergraph $H^{\prime}=\left(V^{\prime}, E^{\prime}\right)$, with $V^{\prime}=V \backslash\{v\}$ and $E^{\prime}=\left\{e \in\left(\begin{array}{c}V^{\prime} \\ k-1\end{array}\right): e \cup\{v\} \in E\right\}$. Following the Example 22, we construct a scheme for the sub-hypergraph determined by all hyperedges adjacent to $v$. Then 
we remove $v$ and its adjacent hyperedges from $H$. We choose another vertex $v^{\prime}$ adjacent to $\ell_{2}>d$ excluded hyperedges and do the same until no vertices with degree greater than $d$ in $\bar{H}$ exist.

Since in the beginning there are $\ell$ excluded hyperedges, and in each step we remove $\ell_{i}>d$ hyperedges, the number of steps is at most $\ell / d$. Thus, the total share size of the resulting scheme is $\tilde{O}\left(2^{k-1}(k-1)^{k-1} n \sum_{i=1}^{\ell / d} \ell_{i}^{1-\varepsilon_{k-1}}\right)$. As $\sum_{i=1}^{\ell / d} \ell_{i} \leq \ell$, the above expression is maximized when $\ell_{1}=\cdots=\ell_{\ell / d}=d$, and the total share size of the scheme is $\tilde{O}\left(2^{k-1}(k-1)^{k-1} n \ell / d^{\varepsilon_{k-1}}\right)$.

Finally, since the degree of $\bar{H}$ at most $d$, we use Lemma 20 to construct a secret-sharing scheme realizing $H$ with total share size $\tilde{O}\left(2^{k} k^{k} d^{k-1} n\right)$.

Corollary 24. Let $H=(V, E)$ be a k-hypergraph with $|V|=n$ and $|E|=\left(\begin{array}{l}n \\ k\end{array}\right)-\ell$ for some $\ell k<n$. There exists a secret-sharing scheme realizing $H$ with total share size $n+\tilde{O}\left(2^{k} k^{k+2} \ell^{2}\right)$.

Proof. Define $W \subseteq V$ as the set of vertices of degree zero in $\bar{H}$. Since $\ell k<n$, $|W|>0$. Consider the $k$-hypergraph $H^{\prime}=\left(V, E^{\prime}\right)$ with $E^{\prime}=\left\{e \in\left(\begin{array}{l}V \\ k\end{array}\right): \mid e \cap\right.$ $W \mid \geq 1\}$. Observe that $H^{\prime} \subseteq H$. By [40, there exists an ideal secret-sharing scheme realizing $H^{\prime}$. Now it remains to find a secret-sharing scheme for $H \backslash H^{\prime}$, a hypergraph defined on $V \backslash W$ whose complement has at most $\ell k$ vertices and $\ell$ hyperedges. The proof is completed by using Theorem 23 .

Remark 25. By [27], the scheme constructed in the first step of the proof of Corollary 24 can be constructed over any finite field $\mathbb{F}$ with $|\mathbb{F}|>\left(\begin{array}{c}n+1 \\ k\end{array}\right)$.

\section{Removing Few Authorized Sets from Access Structures}

Our main result (Theorem 18) shows that if we start with the complete graph and remove "few" edges, then the share size required to realize the new graph is not "too big". We then generalize these results to complete homogeneous hypergraphs. In this section we address the effect of removing few authorized sets from an access structure. We first consider arbitrary graph access structures and then consider access structures where the minimal authorized sets are small and for each party we remove few sets containing the party (this generalizes the case where the complement graph has constant degree). Due to space constraints, the proofs in this section will appear in the full version of this paper.

Theorem 26. Let $G=(V, E)$ and $G^{\prime}=\left(V, E^{\prime}\right)$ be two graphs with $E^{\prime} \subset E$, $\left|E \backslash E^{\prime}\right|=\ell$, and $|V|=n$. Assume $G$ can be realized by a scheme in which the total share size is $m$ (clearly, $m \leq n^{2}$ ). If $\ell>m / n$, then $G^{\prime}$ can be realized by a scheme in which the total share size is $\tilde{O}(\sqrt{\ell m n})$. If $\ell \leq m / n$, then $G^{\prime}$ can be realized by a scheme in which the total share size is $m+2 \ell n \leq 3 m$.

In the interesting case in Theorem 26 when $\ell>m / n$, the total share size is $\tilde{O}(\sqrt{\ell m n})$. This is better than the trivial scheme giving shares of total size $O\left(n^{2}\right)$ only when $\ell$ is not to large, namely, $\ell \ll n^{3} / m$. 
We next consider removing authorized sets from more general access structures. We say that access structure $\Gamma$ is of degree $d$ if for every $p \in P$ there are at most $d$ subsets in min $\Gamma$ containing $p$.

Theorem 27. Let $\Gamma_{1}$ and $\Gamma_{2}$ be two access structures on $P$ with $\min \Gamma_{2} \subset$ $\min \Gamma_{1}$ satisfying that $|A| \leq k$ for every $A \in \min \Gamma_{1}$. If $\Gamma_{2}$ is of degree $d$ and there exists a scheme realizing $\Gamma_{1}$ with total share size $m$, then the access structure determined by $\min \Gamma_{1} \backslash \min \Gamma_{2}$ can be realized by a secret-sharing scheme with total share size $\tilde{O}\left(2^{k} k^{k} d^{k-1} m\right)$.

Observe that if $k \ll n$, the removal of minimal authorized subsets from an access structure does not increase so much the share size. Therefore, for $k \ll n$, access structures close to an access structure realized by an efficient scheme are not "hard".

\section{Lower Bounds for Very Dense Graphs}

In this section we show lower bounds on the total share size for realizing very dense graphs. Recall that the best lower bound on the total share size for realizing a graph is $\Omega(n \log n)$ [251021] and the best lower bound on the total share size for realizing a graph by a linear scheme is $\Omega\left(n^{3 / 2}\right)$ [6]. However, these lower bounds use sparse graphs with $\Theta(n \log n)$ and $\Omega\left(n^{3 / 2}\right)$ edges respectively. In this section we will show how to use these sparse graphs to prove lower bounds for very dense graphs. In particular, we show that there exists a graph with $n^{1+\beta}$ excluded edges such that in every linear secret-sharing realizing it, the total share size is $\Omega\left(n^{1+\beta / 2}\right.$ ) (for every $0 \leq \beta<1$ ). This lower bound shows that the total share size grows as a function of $\beta$. However, there is still a gap between our upper and lower bounds. We start with a lower bound for graphs with less than $n$ excluded edges.

Theorem 28. For every $n$ and every $2<\ell<n / 2$, there exists a graph with $n$ vertices and $\ell$ excluded edges such that the total share size of every secret-sharing realizing it is at least $n+\ell$.

Proof. We construct a graph $G=(V, E)$ with $n \geq 2 \ell+1$ vertices. We denote the vertices of the graph by $V=\left\{a, b_{0}, \ldots, b_{\ell-1}, c_{0}, \ldots, c_{\ell-1}, v_{2 \ell+2}, \ldots, v_{n}\right\}$. The graph $G$ has all edges except for the following $\ell$ excluded edges: $\bar{E}=$ $\left\{\left(a, c_{i}\right): 0 \leq i \leq \ell-1\right\}$.

For every $0 \leq i \leq \ell-1$, consider the graph $G$ restricted to the vertices $a, b_{i}, c_{i}, c_{(i+1) \bmod \ell}$. This graph has two excluded edges $\left(a, c_{i}\right)$ and $\left(a, c_{(i+1)} \bmod \ell\right)$. Blundo et al. [11] proved that in any secret-sharing realizing this graph, the sum of the sizes of the shares of $b_{i}$ and $c_{i}$ is at least 3 times the size of the secret. Thus, in any secret-sharing realizing $G$, the sum of the sizes of the shares of $b_{i}$ and $c_{i}$ is at least 3 times the size of the secret. By [35], the size of the share of each party in any secret-sharing realizing any graph with no isolated vertices is at least the size of the secret. Thus, the total share size in any secret-sharing realizing $G$ is at least $n+\ell$. 
Theorem 29. For every $\beta$, where $0 \leq \beta<1$, there exists a graph with $n$ vertices and less than $n^{1+\beta}$ excluded edges, such that the total share size in any linear secret-sharing realizing it is $\Omega\left(n^{1+\beta / 2}\right)$.

Proof. By 6], for every $n$ there exists a graph with $n$ vertices such that the total share size in any linear secret-sharing realizing it is $\Omega\left(n^{3 / 2}\right)$. We use this graph to construct a dense graph $G=(V, E)$ with $n$ vertices. We partition the vertices of $G$ into $n^{1-\beta}$ disjoint sets of vertices $\mathcal{V}_{1}, \ldots, \mathcal{V}_{n^{1-\beta}}$, where $\left|\mathcal{V}_{i}\right|=n^{\beta}$ for $1 \leq i \leq n^{1-\beta}$. We construct the edges as follows: First, for every 2 vertices $u$ and $v$ such that $u \in \mathcal{V}_{i}$ and $v \in \mathcal{V}_{j}$ for $i \neq j$, we add the edge $(u, v)$ to $E$, i.e., there is an edge connecting every 2 vertices from different parts. Second, for every $i$ (where $1 \leq i \leq n^{1-\beta}$ ), we construct a copy of the graph from [6] with $n^{\beta}$ vertices among the vertices of $\mathcal{V}_{i}$. We denote this graph by $G_{\mathcal{V}_{i}}$.

Since all excluded edges in the above construction are between vertices in the same part, the number of excluded edges is at most $\left(\begin{array}{c}n^{\beta} \\ 2\end{array}\right) n^{1-\beta}<n^{1+\beta}$. The total share size of any linear secret-sharing scheme realizing $G_{\mathcal{V}_{i}}$ (for $1 \leq i \leq n^{1-\beta}$ ) is $\Omega\left(\left(n^{\beta}\right)^{3 / 2}\right)=\Omega\left(n^{3 \beta / 2}\right)$. Thus, the total share size of any linear secret-sharing scheme realizing $G$ is at least $\Omega\left(n^{1-\beta} n^{3 \beta / 2}\right)=\Omega\left(n^{1+\beta / 2}\right)$.

Theorem 30. For every $\beta$, where $0<\beta<1$, there exists a graph with $n$ vertices and less than $n^{1+\beta}$ excluded edges such that the share size of any secret-sharing scheme realizing it is $\Omega(\beta n \log n)$.

Proof. We use the construction from the proof of Theorem 29, where for every $1 \leq i \leq n^{1-\beta}$ we set $G_{\mathcal{V}_{i}}$ to be a $\log n^{\beta}$-dimensional cube. By 21 , any secretsharing scheme realizing $G_{\mathcal{V}_{i}}$ has a total share size of $\Omega\left(\beta n^{\beta} \log n\right)$. Thus, any secret-sharing scheme realizing $G$ must have a total share size of $\Omega\left(\left(n^{1-\beta}\right)\right.$. $\left.\left.\beta n^{\beta} \log n\right)\right)=\Omega(\beta n \log n)$.

Acknowledgment. We thank Noga Alon and Stasys Jukna for discussions on equivalence covering, and Ilan Orlov for useful discussions and suggestions.

The second author's work is partly supported by the Spanish Government through projects TIN201127076-C03-01, 2010 CSD2007-00004, and by the Catalan Government through grant 2009 SGR 1135.

\section{References}

1. Alon, N.: Covering graphs by the minimum number of equivalence relations. Combinatorica 6(3), 201-206 (1986)

2. Alon, N., Spencer, J.H.: The Probabilistic Method, 3rd edn. John Wiley \& Sons (2008)

3. Babai, L., Gál, A., Wigderson, A.: Superpolynomial lower bounds for monotone span programs. Combinatorica 19(3), 301-319 (1999)

4. Beimel, A.: Secret-Sharing Schemes: A Survey. In: Chee, Y.M., Guo, Z., Ling, S., Shao, F., Tang, Y., Wang, H., Xing, C. (eds.) IWCC 2011. LNCS, vol. 6639, pp. 11-46. Springer, Heidelberg (2011) 
5. Beimel, A., Chor, B.: Universally ideal secret sharing schemes. IEEE Trans. on Information Theory 40(3), 786-794 (1994)

6. Beimel, A., Gál, A., Paterson, M.: Lower bounds for monotone span programs. Computational Complexity 6(1), 29-45 (1997)

7. Ben-Or, M., Goldwasser, S., Wigderson, A.: Completeness theorems for noncryptographic fault-tolerant distributed computations. In: 20th STOC, pp. 1-10 (1988)

8. Benaloh, J., Leichter, J.: Generalized Secret Sharing and Monotone Functions. In: Goldwasser, S. (ed.) CRYPTO 1988. LNCS, vol. 403, pp. 27-35. Springer, Heidelberg (1990)

9. Blakley, G.R.: Safeguarding cryptographic keys. In: 1979 AFIPS National Computer Conference, pp. 313-317 (1979)

10. Blundo, C., De Santis, A., de Simone, R., Vaccaro, U.: Tight bounds on the information rate of secret sharing schemes. Des. Codes Cryptogr. 11(2), 107-122 (1997)

11. Blundo, C., De Santis, A., Gargano, L., Vaccaro, U.: On the information rate of secret sharing schemes. Theoretical Computer Science 154(2), 283-306 (1996)

12. Blundo, C., De Santis, A., Stinson, D.R., Vaccaro, U.: Graph decomposition and secret sharing schemes. J. of Cryptology 8(1), 39-64 (1995)

13. Brickell, E.F.: Some ideal secret sharing schemes. Journal of Combin. Math. and Combin. Comput. 6, 105-113 (1989)

14. Brickell, E.F., Davenport, D.M.: On the classification of ideal secret sharing schemes. J. of Cryptology 4(73), 123-134 (1991)

15. Bublitz, S.: Decomposition of graphs and monotone formula size of homogeneous functions. Acta Informatica 23, 689-696 (1986)

16. Capocelli, R.M., De Santis, A., Gargano, L., Vaccaro, U.: On the size of shares for secret sharing schemes. J. of Cryptology 6(3), 157-168 (1993)

17. Chaum, D., Crépeau, C., Damgård, I.: Multiparty unconditionally secure protocols. In: 20th STOC, pp. 11-19 (1988)

18. Chor, B., Kushilevitz, E.: Secret sharing over infinite domains. J. of Cryptology 6(2), 87-96 (1993)

19. Cramer, R., Damgård, I., Maurer, U.: General Secure Multi-party Computation from any Linear Secret-Sharing Scheme. In: Preneel, B. (ed.) EUROCRYPT 2000. LNCS, vol. 1807, pp. 316-334. Springer, Heidelberg (2000)

20. Di Crescenzo, G., Galdi, C.: Hypergraph decomposition and secret sharing. Discrete Applied Mathematics 157(5), 928-946 (2009)

21. Csirmaz, L.: Secret sharing schemes on graphs. Cryptology ePrint Archive, 2005/059 (2005)

22. Csirmaz, L.: An impossibility result on graph secret sharing. Des. Codes Cryptog. 53(3), 195-209 (2009)

23. Csirmaz, L., Tardos, G.: Secret sharing on trees: problem solved. Cryptology ePrint Archive, 2009/71 (2009)

24. Desmedt, Y., Frankel, Y.: Shared Generation of Authenticators and Signatures. In: Feigenbaum, J. (ed.) CRYPTO 1991. LNCS, vol. 576, pp. 457-469. Springer, Heidelberg (1992)

25. van Dijk, M.: On the information rate of perfect secret sharing schemes. Des. Codes and Cryptog. 6, 143-169 (1995)

26. Erdös, P., Pyber, L.: Covering a graph by complete bipartite graphs. Discrete Mathematics 170(1-3), 249-251 (1997)

27. Farràs, O., Martí-Farré, J., Padró, C.: Ideal multipartite secret sharing schemes. J. of Cryptology 25(1), 434-463 (2012)

28. Gál, A.: A characterization of span program size and improved lower bounds for monotone span programs. In: 30th STOC, pp. 429-437 (1998) 
29. Gál, A., Pudlák, P.: Monotone complexity and the rank of matrices. IPL 87, 321326 (2003)

30. Goyal, V., Pandey, O., Sahai, A., Waters, B.: Attribute-based encryption for finegrained access control of encrypted data. In: 13th CCS, pp. 89-98 (2006)

31. Ito, M., Saito, A., Nishizeki, T.: Secret sharing schemes realizing general access structure. In: Globecom 1987, pp. 99-102 (1987)

32. Jerrum, M.: A very simple algorithm for estimating the number of k-colorings of a low-degree graph. Random Structures \& Algorithms 7, 157-166 (1995)

33. Jukna, S.: On set intersection representations of graphs. Journal of Graph Theory $61,55-75(2009)$

34. Karchmer, M., Wigderson, A.: On span programs. In: 8th Structure in Complexity Theory, pp. 102-111 (1993)

35. Karnin, E.D., Greene, J.W., Hellman, M.E.: On secret sharing systems. IEEE Trans. on Information Theory 29(1), 35-41 (1983)

36. Martí-Farré, J., Padró, C.: Secret sharing schemes on sparse homogeneous access structures with rank three. Electr. J. Comb. 11(1) (2004)

37. Martí-Farré, J., Padró, C.: Secret sharing schemes with three or four minimal qualified subsets. Des. Codes Cryptog. 34(1), 17-34 (2005)

38. Martí-Farré, J., Padró, C.: On secret sharing schemes, matroids and polymatroids. Journal of Mathematical Cryptology 4(2), 95-120 (2010)

39. Naor, M., Wool, A.: Access control and signatures via quorum secret sharing. In: 3rd CCS, pp. 157-167 (1996)

40. Padró, C., Sáez, G.: Secret sharing schemes with bipartite access structure. IEEE Trans. on Information Theory 46, 2596-2605 (2000)

41. Padró, C., Sáez, G.: Lower bounds on the information rate of secret sharing schemes with homogeneous access structure. IPL 83(6), 345-351 (2002)

42. Salas, J., Sokal, A.D.: Absence of phase transition for antiferromagnetic Potts models via the Dobrushin uniqueness theorem. J. Statist. Phys. 86, 551-579 (1997)

43. Shamir, A.: How to share a secret. Communications of the ACM 22, 612-613 (1979)

44. Shankar, B., Srinathan, K., Pandu Rangan, C.: Alternative Protocols for Generalized Oblivious Transfer. In: Rao, S., Chatterjee, M., Jayanti, P., Murthy, C.S.R., Saha, S.K. (eds.) ICDCN 2008. LNCS, vol. 4904, pp. 304-309. Springer, Heidelberg (2008)

45. Simmons, G.J., Jackson, W., Martin, K.M.: The geometry of shared secret schemes. Bulletin of the ICA 1, 71-88 (1991)

46. Stinson, D.R.: New General Lower Bounds on the Information Rate of Secret Sharing Schemes. In: Brickell, E.F. (ed.) CRYPTO 1992. LNCS, vol. 740, pp. 168-182. Springer, Heidelberg (1993)

47. Stinson, D.R.: Decomposition construction for secret sharing schemes. IEEE Trans. on Information Theory 40(1), 118-125 (1994)

48. Sun, H., Shieh, S.: Secret sharing in graph-based prohibited structures. In: INFOCOM, pp. 718-724 (1997)

49. Sun, H.-M., Wang, H., Ku, B.-H., Pieprzyk, J.: Decomposition construction for secret sharing schemes with graph access structures in polynomial time. SIAM J. Discret. Math. 24, 617-638 (2010)

50. Tassa, T.: Generalized oblivious transfer by secret sharing. Des. Codes Cryptog. 58(1), 11-21 (2011)

51. Waters, B.: Ciphertext-Policy Attribute-Based Encryption: An Expressive, Efficient, and Provably Secure Realization. In: Catalano, D., Fazio, N., Gennaro, R., Nicolosi, A. (eds.) PKC 2011. LNCS, vol. 6571, pp. 53-70. Springer, Heidelberg (2011)

52. Wegener, I.: The Complexity of Boolean Functions. Wiley-Teubner (1987) 